\title{
The formation of super-star clusters in disk and dwarf galaxies
}

\author{
Carsten Weidner $^{1}$, Ian A. Bonnell ${ }^{1}$ and Hans Zinnecker ${ }^{2,3}$ \\ ${ }^{1}$ SUPA, School of Physics \& Astronomy, University of St Andrews, North Haugh, St \\ Andrews, Fife KY16 9SS, UK \\ email: cw60@st-andrews.ac.uk, iab1@st-andrews.ac.uk \\ ${ }^{2}$ Astrophysikalisches Institut Potsdam, An der Sternwarte 16, D-14482 Potsdam, Germany \\ ${ }^{3}$ SOFIA Science Center, University of Stuttgart, Institut für Raumfahrtsyteme, \\ Pfaffenwaldring 31, D-70569 Stuttgart, Germany \\ email: zinnecker@dsi.uni-stuttgart.de
}

\begin{abstract}
Super-star clusters are probably the largest star-forming entities in our local Universe, containing hundreds of thousands to millions of young stars usually within less than a few parsecs. While no such systems are known in the Milky Way (MW), they are found especially in pairs of interacting galaxies but also in some dwarf galaxies like R 136 in the Large Magelanic Cloud (LMC). With the use of SPH calculations we show that a natural explanation for this phenomenon is the presence of shear in normal spiral galaxies which facilitates the formation of low-density loose OB associations from giant molecular clouds (GMC) instead of dense super-star clusters. In contrast, in interacting galaxies and in dwarf galaxies, regions can collapse without having a large-scale sense of rotation. This lack of rotational support allows the giant molecular clouds to concentrate into a single, dense and gravitationally bound system.
\end{abstract}

Keywords. ISM: clouds - open clusters and associations: general - galaxies: star clusters: general - galaxies: star formation

\section{Introduction}

The formation of stars does not take place in isolation but in loose groups or embedded clusters in Giant Molecular Clouds, each cluster containing a dozen to many million of stars (Lada \& Lada 2003). Such embedded clusters need not be bound structures and actually $\sim 90 \%$ of them will dissolve within about 10 Myr (Lada \& Lada 2003) due to gas expulsion and internal $N$-body effects.

Interestingly, many so-called super-star clusters $\left(M_{\text {cluster }} \geqslant 10^{5} M_{\odot}\right)$ are not found in star-forming disk galaxies but in relatively small dwarf galaxies. Especially, when excluding merger and interacting galaxies, there seems to be a trend with galaxy type and mass of the most-massive young ( $<50$ Myr) star cluster (Fig. 1).

Besides the total mass, one main difference between dwarf galaxies, disk galaxies and interaction regions of galaxies is the amount of shear acting on GMCs in them. Disk galaxies are rotationally supported systems, resulting in relatively large shear forces on GMCs. Dwarf galaxies show far less amounts of shear. Interacting galaxies with tidally driven structure also have low shear in the tidal arms and the interaction region.

The presence of retrograde rotating GMCs in spiral galaxies is sometimes seen as evidence that the formation and evolution of GMCs is not or only weakly connected to the shear/rotation of spiral arms. But Dobbs (2008) has shown that spiral shock models of GMC formation produce both prograde and retrograde rotating GMCs due to the random nature of the coagulation process. 


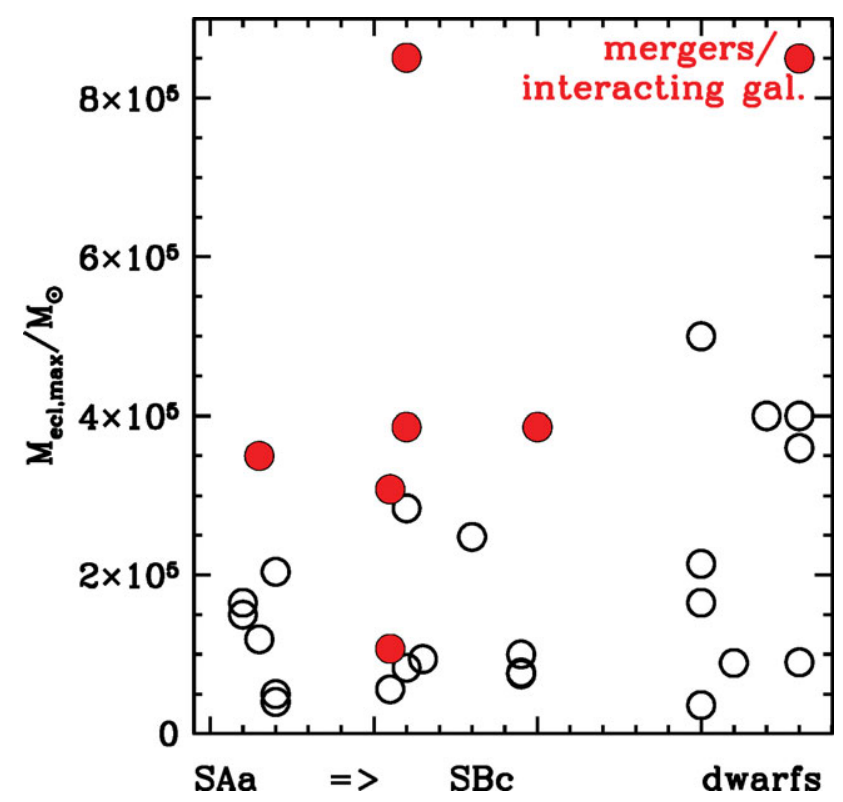

Figure 1. Dependence of the most-massive young cluster in galaxies on the galaxy type.

\section{The Model}

The simulations of the gravitational collapse of GMCs were carried out using a 3DSPH code. The thermal and turbulent energies are initially significantly subvirial and therefore the clouds collapse rapidly due to gravitation. The initial temperature of the gas is about $100 \mathrm{~K}$ and the gas is allowed to cool down to $\approx 10 \mathrm{~K}$ due to a Larson equation-of-state with $\gamma=0.75$ (Larson 2005). $10^{6}$ SPH-particles are used to model the $10^{6} M_{\odot}$ gas within $50 \mathrm{pc}$ cloud radius. Star formation is modelled by sink particles, which can grow through accretion of infalling gas ( $\mathrm{SPH}$ particles) and interact gravitationally with the rest of the simulation.

The calculations are evolved for about one free-fall time of the GMC. With a radius of $R_{\mathrm{GMC}}=50 \mathrm{pc}$ and a mass $M_{\mathrm{GMC}}=10^{6} M_{\odot}$ the free-fall time is $t_{\mathrm{ff}}=5.9 \mathrm{Myr}$ in all cases considered here.

Four different models are calculated with identical initial conditions but four different levels of shear, corresponding to solid body rotation with angular velocities of $\Omega=0$, $\Omega=2 \times 10^{-15}, 5 \times 10^{-15}$ and $10^{-14} \mathrm{rad} \mathrm{s}^{-1}$.

For MW GMCs rotations rates of $\Omega_{\mathrm{MW}} \sim 3-6 \times 10^{-15} \mathrm{rad} \mathrm{s}^{-1}$ are observed (Bissantz et al. 2003) while for the LMC the rate is significantly lower with $\Omega_{\mathrm{LMC}} \sim$ $6 \times 10^{-16} \mathrm{rad} \mathrm{s}^{-1}$ (Alves \& Nelson 2000).

Models 2-4 represent typical conditions expected in spiral galaxies.

The simulations presented here do not include any feedback from supernovae, radiation or stellar winds. Though, the effects of ionizing radiation (Dale et al. 2005) and stellar winds (Dale \& Bonnell 2008) have been studied before. We note that the inclusion of these sources of feedback in the above models did not have a significant effect on the star formation rate or efficiency. 


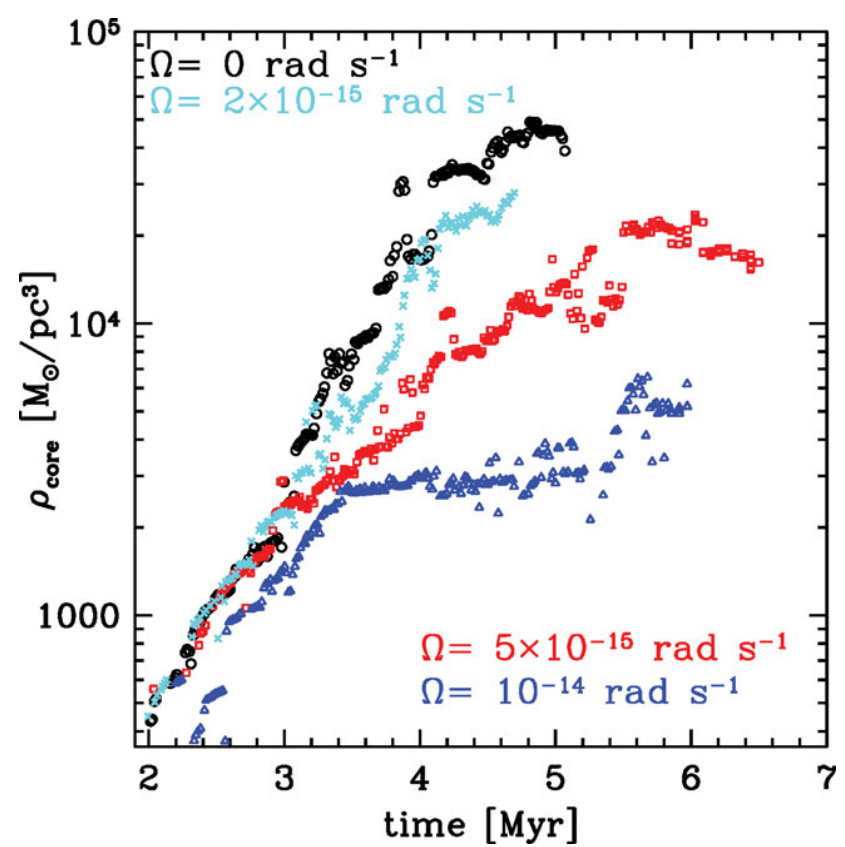

Figure 2. Temporal evolution of mass density in a core with $r_{\text {core }}=1.0 \mathrm{pc}$. The black dots mark model 1 , the turquoise crosses model 2 , the red boxes model 3 and the blue triangles model 4. The final densities are about 3 to 15 times larger for the no shear run than those in the runs with shear.

\section{Results}

In the models with low or no shear, more sinks are formed and they are much more highly concentrated towards the center of the cloud. In Figure 2 is shown how the density of mass in sinks evolves with time. The mass density is calculated in a sphere of $r_{\text {core }}$ $=1.0 \mathrm{pc}$ around the center of mass of all sinks. After about $3 \mathrm{Myr}$ the models start to diverge as rotational support in the shear models halts the central collapse while in the absence of shear the formation of a dense core occurs. The no-shear model reaches average central densities in excess of $6 \times 10^{4} M_{\odot} \mathrm{pc}^{-3}$, up to 15 times larger than the runs with shear.

An other indicator for the difference between models with and without shear can be seen in Figure 3. Here the radial distribution of the mass in sinks is shown at a time of 4.3 Myr. In the model without shear, a cluster forms with nearly $10^{5} M_{\odot}$ inside $0.1 \mathrm{pc}$ whereas the models with shear have a much more distributed population and much less significant central condensations. Though the total stellar masses formed in all four cases does not differ drastically between the models. Therefore the star-formation efficiencies $\left(\mathrm{SFE}=M_{\mathrm{sinks}} / M_{\mathrm{GMC}}\right)$ are relatively similar, with values between 20 and $40 \%$. It is simply the distribution of the resultant stellar populations which are very different.

\section{Conclusions}

The models show that the formation of super-star clusters depends crucially on the shear content in the pre-collapse giant molecular cloud. Massive clouds in slowly and non rotating environments, as to be expected in dwarf galaxies like the LMC and in interaction regions of colliding galaxies, tend to form a massive, centrally condensed cluster, in agreement with the results by Escala \& Larson (2008). GMC's in disk galaxies 


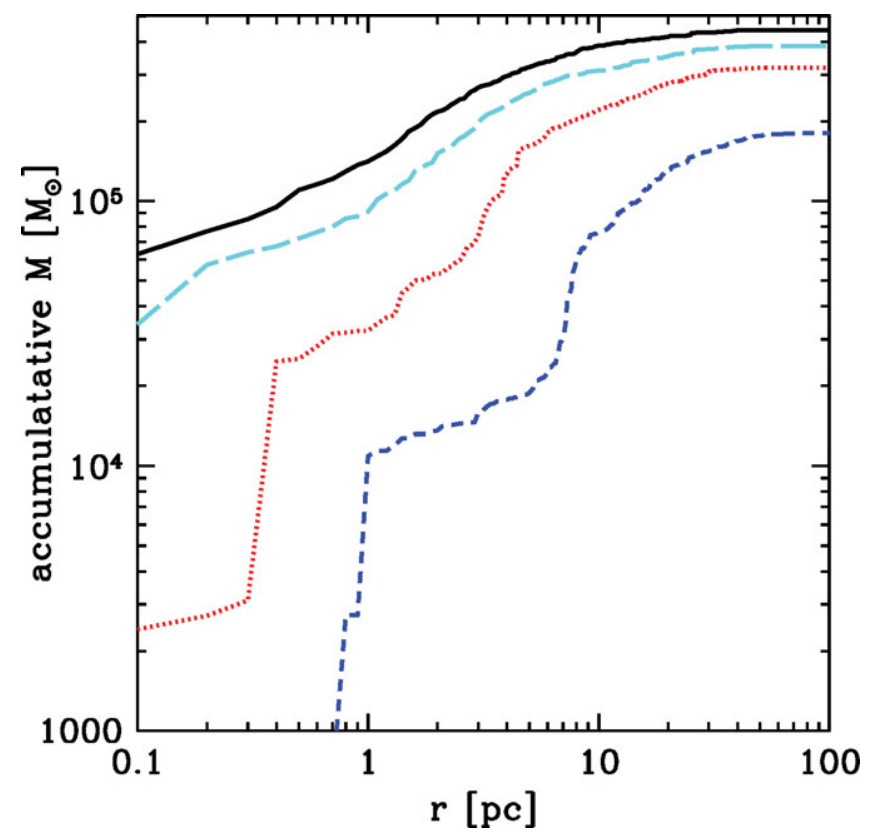

Figure 3. The radial dependence of the accumulated mass for all four simulations after $4.3 \mathrm{Myr}$ for the no shear model (solid black line), the light shear model (long-dashed turquoise line), the intermediate shear one (dotted red line) and the high shear model (dashed blue line).

are more likely to fragment and form a system of smaller clusters or structures more like OB associations. Globular clusters are therefore unlikely to have formed in the disk of galaxies. They may originate from an initial monolithic collapse which formed the bulge of a galaxy, a major merging event, or they have formed in dwarf galaxies which have then been accreted later on (Zinnecker et al. 1988).

\section{References}

Alves, D. R. \& Nelson, C. A. 2000, ApJ, 542, 789

Bissantz, N., Englmaier, P., \& Gerhard, O. 2003, MNRAS, 340, 949

Dale, J. E. \& Bonnell, I. A. 2008, MNRAS, 391, 2

Dale, J. E., Bonnell, I. A., Clarke, C. J., \& Bate, M. R. 2005, MNRAS, 358, 291

Dobbs, C. L. 2008, MNRAS, 391, 844

Escala, A. \& Larson, R. B. 2008, ApJ, 685, L31

Lada, C. J. \& Lada, E. A. 2003, ARA\&A, 41, 57

Larson, R. B. 2005, MNRAS, 359, 211

Zinnecker, H., Keable, C. J., Dunlop, J. S., Cannon, R. D., \& Griffiths, W. K. 1988, in IAU Symposium, Vol. 126, The Harlow-Shapley Symposium on Globular Cluster Systems in Galaxies, ed. J. E. Grindlay \& A. G. D. Philip, 603-+ 